\title{
Eqüidade e Reforma em Sistemas de Serviços de Saúde: o caso do SUS
}

\section{Equity and Reform in Health Services System: the case of Unified Health System of Brazil}

Jairnilson Silva Paim

Professor Titular do Instituto de Saúde Coletiva da Universidade

Federal da Bahia. Pesquisador I-A do CNPq.

E-mail: jairnilळufba.br

\section{Resumo}

O ensaio procurou responder a seguinte pergunta: o SUS é uma política pública de promoção da eqüidade? Nesse sentido, apresenta alguns delineamentos prévios sobre certas noções presentes na pergunta, especialmente sobre as concepções de eqüidade e do SUS. Realiza uma breve revisão sobre reformas setoriais em contraponto com a Reforma Sanitária Brasileira e um sumário do perfil de desigualdades em saúde no país. Conclui examinando alguns esforços para a redução dessas desigualdades levantando a hipótese de que o SUS pode promover eqüidade no sentido de justiça sem comprometer o seu caráter universal e igualitário.

Palavras-chave: Eqüidade; Política pública; Política de saúde; Sistema de saúde; Sistema Único de Saúde. 


\section{Abstract}

This paper aimed to answer the following question: Is SUS a public policy of equity promotion? In this direction, it presents previous thoughts on some terms, especially on the concepts of equity and on SUS. A brief review is carried out on sector's reforms in order to discuss the Brazilian Sanitary Reform. Also, a summary of health inequalities profile in the country is given. It concludes by examining some efforts for the reduction of these inequalities by formulating the hypothesis that SUS can promote equity in the direction of justice without compromising its universal and egalitarian character.

Keywords: Equity; Public Policy; Health Policy; Health System; Unified Health System of Brazil.

\section{Introdução}

As reformas nos sistemas de serviços de saúde na América Latina têm sido analisadas considerando-se doze eixos temáticos: marco jurídico; direito aos cuidados de saúde; aumento da cobertura; função reitora dos Ministérios da Saúde; descentralização; participação e controle social; oferta de serviços; separação de funções, modelo de gestão; recursos humanos; qualidade; avaliação de tecnologias. Na avaliação dos seus resultados têm-se tomado como atributos a eqüidade, eficiência, efetividade, sustentabilidade, qualidade, participação e controle social (Infante e col., 200o).

Paradoxalmente - num contexto internacional em que as desigualdades entre países, regiões, classes e grupos sociais e étnico-raciais mais se avolumam, em decorrência da reestruturação produtiva e das políticas econômicas impostas pelos países centrais do capitalismo globalizado e pelos organismos internacionais multilaterais (Soares, 1999; Cordeiro, 2001; Elias, 2005), vinculados aos seus interesses - essas mesmas organizações recorrem à retórica da eqüidade, como tentativa de mascarar as iniqüidades que contribuíram para produzir.

No Brasil, este termo vem sendo progressivamente utilizado em documentos técnicos, normativos e doutrinários (Kadt e Tasca, 1993), inclusive nos relatórios das conferências nacionais de saúde (Pinheiro e col, 2005). Desde a realização da $11^{\mathrm{a}}$ Conferência Nacional de Saúde, realizada no ano 200o, o Conselho Nacional de Saúde tem formulado proposições e estratégias reunidas em onze compromissos apresentados aos candidatos das eleições presidenciais de 2002, entre os quais "a construção da eqüidade" destaca-se em primeiro lugar (Conselho Nacional de Saúde, 2002, p.297).

Assim, a eqüidade vem sendo reiterada como uma das principais referências para a avaliação das reformas setoriais e para a reorientação de políticas e de sistemas de saúde. Daí a pertinência e oportunidade da pergunta que orientou a elaboração do presente texto: O SUS é uma política pública de promoção da eqüidade? 


\section{Alguns Delineamentos Prévios}

Se a questão acima já é complexa em termos de formulação, mais desafiante, ainda, é a tentativa de respondê-la. No que diz respeito à pergunta, em si, diversas considerações podem ser feitas. De que SUS se está tratando? O que se entende como política pública? Pretende-se privilegiar qual momento do ciclo de uma política pública? Trata-se de uma avaliação implícita do SUS enquanto política pública? Insinuando-se uma avaliação, a ênfase seria na formulação, no processo de implementação, nos produtos, nos resultados, no impacto? Qual o conceito de eqüidade que fundamentaria tal questão? Diante da multiplicidade de possíveis respostas a essas indagações, procurar-se-á proceder a alguns delineamentos prévios para precisar alguns ângulos de análise para fins de elaboração do ensaio.

O Sistema Único de Saúde (SUS) corresponde, nas suas origens, a uma das proposições do documento $A$ questão democrática na área da saúde, apresentado pelo Centro Brasileiro de Estudos de Saúde (Cebes) no $1^{o}$ Simpósio de Política Nacional de Saúde, promovido pela Comissão de Saúde da Câmara dos Deputados em 1979 (Escorel, 1998). Representa uma dimensão setorial e institucional da proposta da Reforma Sanitária Brasileira (RSB), formulada pelo movimento da democratização da saúde (Paim, 1988) e conhecido como movimento sanitário, cujo corpo doutrinário foi sistematizado e consagrado durante a $8^{a}$. Conferência Nacional de Saúde, em 1986. Após as discussões realizadas pela Comissão Nacional de Reforma Sanitária parte daquela proposta foi incorporada à Constituição da República em 1988 e na legislação ordinária em 1990 (Paim, 2003C).

Conseqüentemente, diversas concepções ou "projetos" de SUS passaram a se delinear e competir no imaginário de técnicos, gestores, políticos, dirigentes e população, bem como na arena político-institucional, durante a sua implantação nos últimos 15 anos. Em outras oportunidades tem-se ressaltado pelo menos quatro tipos de concepções: o SUS formal, estabelecido pela Constituição, Lei Orgânica da Saúde e portarias; o SUS democrático, integrante de uma totalidade de mudanças desenhadas pela RSB; o SUS real, com direito a trocadilho com o nome da moeda que simboliza as políticas econômicas monetaristas e de ajuste macro-estrutural, refém da chamada área eco- nômica dos governos e do clientelismo e do patrimonialismo que caracterizam o Estado brasileiro; e o SUS para pobres, como parte das políticas focalizadas defendidas por organismos internacionais. Na medida em que as duas últimas concepções do SUS representariam a sua implantação tortuosa, a análise tomará como "padrão ouro" o SUS formal e o SUS democrático e como evidências empíricas àquelas obtidas na sua implementação tortuosa (Paim, 2003c).

Entende-se como política uma dada distribuição do poder no Estado, na sociedade e instituições. Mais que princípios, diretrizes e planos de ação (policy), as políticas de saúde têm a ver com a distribuição dos poderes técnico (informações), administrativo (recursos) e político (mobilização), no âmbito setorial, e com as formas de apropriação e exercício dos poderes político (força), econômico (riqueza), ideológico (saber) e simbólico (representações) no âmbito societário (politics). Desde que não se confunda o público com o estatal, uma política pública em saúde corresponde às respostas sociais - via Estado, empresas, o chamado "terceiro setor" e comunidade - face às condições de saúde e dos seus determinantes, bem como diante da produção, distribuição e regulação de bens, serviços e ambientes que afetam a saúde das pessoas e das populações (Paim, 2002; 2003c). Tais respostas sociais, portanto, não se restringem à formulação de documentos normativos e planos, ao financiamento nem à realização de ações. Essas respostas, ao mesmo tempo, implicam uma dada distribuição do poder, conforme os tipos acima mencionados, que pode ser acionada para o crescimento, mudança e legitimação.

Considerando-se os momentos do ciclo de uma política pública, pretende-se contemplar, no caso do SUS, os momentos da formulação, da implementação e da avaliação. Com relação à avaliação, pode envolver a concepção e o desenho (formulação), o processo (implementação) e, também, os efeitos (produtos, resultados e impacto), configurando três tipos de avaliação: a) avaliação relacionada a conceitualização e ao desenho; b) monitoramento da implementação; c) estimação e avaliação dos resultados e utilidade (Oxman, 1988; Bustamante e Portales, 1988). Embora o presente ensaio não tenha a pretenção de realizar uma avaliação do SUS, mesmo enquanto política pública, é importante ter esses tipos e momentos como referências para facilitar a reflexão e a discussão. 
Finalmente, cabe considerar a eqüidade como o componente mais delicado da pergunta. Não obstante as suas imprecisões e ambigüidades conceituais, alguns esforços voltados para definição desta noção bem como para a sua fundamentação teórica têm sido empreendidos mais recentemente. No entanto, a sua inclusão na agenda e nas proposições de um conjunto de organismos internacionais, justamente no contexto das políticas de ajuste macro-econômico e de desajuste estrutural (Tavares, 1999), incita alguma reflexão e prudência quanto a sua utilidade no exame de um sistema de serviços de saúde que pretende ser universal e igualitário como o SUS.

\section{Qual Eqüidade?}

Do ponto de vista semântico e etimológico a palavra eqüidade está próxima da igualdade podendo ser considerada como sinônimo. Ambas são consideradas contrapontos para as desigualdades sócio-econômicas e de saúde (Pinheiro et. al. 2005). No caso da saúde, haveria que distinguir inicialmente as necessidades de saúde e as necessidades de serviços de saúde, o que remeteria para a separação, com fins analíticos, entre desigualdades nas condições de vida e saúde, de um lado, e desigualdades no acesso e consumo de serviços de saúde, de outro (Almeida, 2002).

Organismos internacionais da área de saúde admitem que equidade implica: “a) en condiciones de salud, disminuir las diferencias evitables e injustas al mínimo posible; b) en servicios de salud, recibir atención en función de la necesidad (equidad de acceso y uso) y contribuir en función de la capacidad de pago (equidad financiera)" (OPS, 1997:16). Tal entendimento legitima o pagamento de serviços de saúde pelos que podem fazê-lo, justificando e restringindo a atuação dos serviços públicos para os pobres. Inspira-se em um texto muito divulgado pelo Escritório Regional da OMS na Europa (Whitehead, 1990) cuja abordagem de equiidade em saúde contempla "diferenças evitáveis e desnecessárias e que também podem ser consideradas injustas" (Pinheiro col., 2005:451), sugerindo que as desigualdades inaceitáveis e injustas mereceriam correções.

Segundo esses autores o termo eqüidade não aparece na base de dados MEDLINE como descritor e sim na do LILACS, referido-se à cobertura, distribuição e uso de recursos, acesso e estado de saúde. (Pinheiro col., 2005:451). Portanto, embora a maioria dos textos técnicos e doutrinários que tratam da questão da eqüidade não tenha como preocupação uma precisão conceitual, é possível identificar dois enfoques subjacentes que tentam distingui-la da noção de igualdade: o econômico e o da justiça.

No enfoque econômico trata-se de buscar uma distribuição mais eficiente dos recursos, recorrendo a estudos de análise econômica, a modelos matemáticos e estudos econométricos. Tem sido objeto privilegiado da Economia da Saúde, enquanto disciplina acadêmica (Andrade e col., 2004). Parte dos autores realiza incursões reflexivas quanto à justiça distributiva, buscando referências na obra de Amartya Sen, que ganhou o Prêmio Nobel (Sen, 2002). Todavia, no plano das políticas patrocinadas por organismos internacionais, esse enfoque apresenta-se sob uma postura aparente de Robin Hood, defendendo políticas de focalização em contraposição às políticas universais, além da concentração de recursos nos mais necessitados - Pro-Poor Programs - (Soares, 1999), sejam regiões, estados, municípios, distritos, bairros, grupos ou pessoas.

Já o enfoque centrado na idéia de justiça recorre à filosofia de Aristóteles e Platão a Rawls e Sen, passando por Hobbes, Rousseau, Kant, Hume, Marx e Engels - para demonstrar que a igualdade pode não ser justa (Ribeiro, 2005; Siqueira-Batista e Schramm, 2005). Embora no presente texto não caiba uma revisão detalhada deste enfoque, as referências citadas podem indicar a pertinência de aprofundar a reflexão sobre o conceito de eqüidade. Assim, "a noção de eqüidade se associa de modo diverso à igualdade e, sobretudo, à justiça, no sentido de propiciar a correção daquilo em que a igualdade agride e, portanto, naquilo que a justiça deve realizar. Tomada nesse sentido, a eqüidade requer igualdade para produzir efeitos, pois se constitui justamente em corretora da situação de igualdade, na medida em que a adoção deste recurso se revele imperfeita diante dos objetivos da promoção da justiça” (Elias, 2005, p.291). Ao se debruçarem sobre tais princípios e diretrizes, disciplinas como Ética e Política de Saúde passam a devotar certa atenção para a questão da eqüidade. Nessa perspectiva, indaga-se, por exemplo, "em nome de que padrão de justiça social as distintas racionalidades de distribuição de recur- 
sos utilizadas pelas políticas setoriais ou pelos níveis de governo são formuladas?” (Cohn, 2005, p.287).

\section{Reformas Setoriais e RSB}

De um modo geral, as reformas setoriais empreendidas na América Latina e no Caribe ao longo da década de 1990 (Pego e Almeida, 2002; Hernandez, 2002; Labra, 2002; Belmartino, 2002; Mitjavila e col., 2002), sob o patrocínio e direção de organismos internacionais, a exemplo do Banco Mundial e Banco Interamericano de Desenvolvimento, e vinculadas aos pacotes de ajuste macroeconômico, apresentam alguns elementos comuns: modificação de regras de financiamento, participação de agentes públicos e privados e separação das funções de financiamento, provisão e regulação (Almeida, 200o).

Ainda que o Brasil não tenha ficado imune a tais influências, o projeto do SUS foi estruturado antes dessas reformas setoriais. Na realidade, o SUS representava a dimensão institucional de uma proposta mais ampla reconhecida como Reforma Sanitária, cujo movimento social que lhe tem sustentado, política e ideologicamente, marca a especificidade do projeto e do processo brasileiro. A RSB e o SUS, portanto, não têm origem nos organismos internacionais, no Estado Brasileiro, na área econômica de governos, nem mesmo no Ministério da Saúde. Estas conquistas do movimento sanitário são supra-partidárias e estão vinculadas às idéias de democratização da saúde, direitos humanos e cidadania.

Conseqüentemente, a agenda de reformas desses organismos internacionais encontrou certa resistência ou oposição aberta no Brasil, pois contrariava o caráter universal das políticas de saúde projetadas pelo movimento da Reforma Sanitária desde a década de 1970, além de ter ameaçado os preceitos constitucionais e a legislação sanitária. Contudo, muitas das iniciativas desses organismos, voltadas para a descentralização e focalização das ações de saúde e para a segmentação do sistema de saúde, encontraram alguma aceitação (Paim, 2004).

Apesar dos constrangimentos impostos ao desenvolvimento das políticas de saúde, pelo ajuste macroestrutural, o país conseguiu manter os instrumentos legais que asseguram o direito à saúde, particularmente no que se refere à universalização da atenção à saúde (Almeida e col., 2002). Ao contrário de outras políticas sociais que, diante das restrições ao gasto público tornaram-se focalizadas e seletivas, a política de saúde manteve a sua abrangência, talvez "em razão da capacidade de intermediação de interesses na arena setorial” (Costa, 2002:13). Ainda assim, contradições no processo aprofundaram a segmentação do sistema de saúde brasileiro (Noronha e Soares, 2001). Desse modo, a assistência médica supletiva "atua no sentido de acentuar as desigualdades no consumo de serviços de saúde” (Travassos e col., 200o, p.144).

A mera existência dessas modalidades assistenciais do setor privado em saúde produz efeitos simbólicos ao insinuar maior qualidade, amenidade, agilidade e conforto aos pacientes, em contraposição aos serviços públicos. Esse sistema, voltado para sub-especialidades médicas, compromete a eqüidade já que "os recursos necessários para a atenção altamente técnica orientada para a enfermidade competem com aqueles exigidos para oferecer serviços básicos, especialmente para as pessoas que não podem pagar por eles"(Starfield, 2002:21). Além disso, a oferta desordenada de assistência médica ambulatorial desse setor privado dificulta a realização do planejamento e da programação local em saúde e favorece a concentração de atendimentos a uns indivíduos em detrimento de outros (Paim, 2003b).

Se forem consideradas a competência legal das conferências nacionais de saúde e a sua composição por usuários e trabalhadores de saúde, integrantes da sociedade civil, e por prestadores, representados por empresários e gestores vinculados ao Estado (níveis federal, estadual e municipal) poder-se-ia obter indicações de como a sociedade e o Estado brasileiros tratam a questão da eqüidade no sistema de saúde brasileiro e no SUS, em particular. Na análise dos conteúdos das proposições que utilizavam o termo eqüidade nos relatórios das $9^{\mathrm{a}}, 10^{\mathrm{a}}$ e $11^{\mathrm{a}}$ Conferências Nacionais de Saúde verificou-se o seu aparecimento duas, oito e vinte e cinco vezes, respectivamente (Pinheiro e col., 2005).

O relatório final da $9^{\text {a }}$ CNS não apontava, na análise da situação de saúde, disparidades entre os grupos sociais e assim não especificava os grupos para os quais se dirigia uma dada proposição nem a demanda que levaria a redução da desvantagem. No caso da $10^{a}$., não foi encontrado capítulo com descrição do 
quadro da saúde da população ainda que fosse defendido o fortalecimento do papel do Estado enquanto promotor da eqüidade e especificado um grupo em desvantagem (portadores de deficiência auditiva), para os quais dever-se-ia preparar profissionais aptos para proporcionar-lhes oportunidades mais equânimes. No que diz respeito à $11^{\mathrm{a}}$, embora o termo equidade tenha aparecido mais vezes, "não se encontra um avanço na utilização do mesmo como um critério para elaboração de propostas, predominando ainda avaliações excessivamente vagas e subjetivas" (Pinheiro e col., 2005:456). A eqüidade aparece como princípio reiterado, junto à integralidade e à humanização, como objetivos para a consolidação do SUS, aparentemente entendida como sinônimo de igualdade.

Diante do tratamento incompleto do tema e da não identificação de grupos sociais desfavorecidos e das suas necessidades de saúde, os autores admitem "uma resistência inicial à incorporação do conceito, como um critério para a elaboração de diretrizes de políticas públicas" (Pinheiro e col., 2005, p.457). Se os autores estendessem a análise até a $12^{\text {a }}$ CNS talvez se surpreendessem com uma situação inversa: a excessiva especificação de grupos sociais e de demandas, particularmente no que concerne à organização de serviços, capaz de comprometer a integralidade da atenção e de contribuir para o retrocesso dos programas especiais, verticalizados e focalizados.

\section{Perfil de Desigualdades}

Há mais de um quarto de século vêm sendo realizados no Brasil estudos epidemiológicos apontando as desigualdades na distribuição espacial de mortes e doenças, bem como suas relações com as condições de vida. Portanto, muito antes de o tema ser objeto de comissões, redes e grupos de pesquisas internacionais, a saúde coletiva brasileira já produzia conhecimentos sobre o tema, capaz de fundamentar a reorientação de políticas públicas, econômicas e sociais. Daí a universalidade, a igualdade e a eqüidade representarem grandes desafios para a concretização numa sociedade extremamente desigual como a brasileira. As desigualdades étnico-raciais na saúde, no entanto, só mais recentemente têm sido colocadas na agenda pelos movimentos sociais, negros e indígenas. Assim, requerem mais investigações no sentido de orientar os formuladores de políticas públicas de saúde (Heringer, 2002).

Questões como raça, racismo e saúde têm estado presentes na produção acadêmica mundial. Assim, no banco de referências bibliográficas Web Science, a média anual de artigos publicados indexados por "racismo" aumentou nos últimos qüinqüênios: 159 (19901994), 286 (1995-1999), e 321 (2000-2004) (Faerstein, 2005). No Brasil tal preocupação já tem estado presente em Congressos da Abrasco, na $12^{a}$ Conferência $\mathrm{Na}$ cional de Saúde Sérgio Arouca, em seminários específicos, além de iniciativas governamentais, a exemplo da introdução do quesito cor nos registros do IBGE e nos dos bancos de dados de mortalidade e de nascidos vivos (Chor e Lima, 2005). A produção acadêmica recente em sociologia, economia e demografia aponta as desvantagens da população negra quanto ao emprego, renda, habitação e educação e suas repercussões na saúde como esperança de vida ao nascer, mortalidade por causas externas (Lopes, 2005) e mortalidade infantil (Cardoso e col., 2005).

Os indígenas e as pessoas de cor preta morrem mais cedo e os piores indicadores de mortalidade por causas evitáveis são apresentados por tais grupos. A esperança de vida ao nascer de povos indígenas era no ano 2000 1,3 ano inferior à dos negros, 7,42 anos à dos brancos e 9,18 dos amarelos. Em 2002, a mortalidade infantil das crianças de cor preta superou em até $80 \%$ a mortalidade infantil das brancas (Lopes, 2005). Pretos e pardos morrem duas vezes mais por agressões do que brancos, predominando entre aqueles as doenças cerebrovasculares. A mortalidade materna e por doença hipertensiva e por diabetes mellitus é maior entre as mulheres negras. Ainda que apenas $7 \%$ do total da variação genética humana seja encontrada entre as raças e que um pequeno número de diferenças genéticas, relacionados a condições de saúde, tenha sido identificado entre os grupos étnico-raciais, a raça tem sido considerada um importante "constructo social, que determina identidades, acesso a recursos e a valorização da sociedade (...) interagindo com outros marcadores de posição social (exemplo: gênero, educação, renda)" (Chor e Lima, 2005: 1587). Contribui, desse modo, para a exposição a distintos riscos à saúde. Concluem as autoras que "em sociedades como a 
brasileira, na qual relações de classe são racializadas e relações raciais são dependentes da classe social, a pesquisa epidemiológica deve elucidar o impacto, na saúde, das desigualdades sócio-econômicas e raciais" (Chor e Lima, 2005:1593).

No que se refere à distribuição de recursos da infraestrutura do sistema de serviços de saúde no Brasil, acesso e utilização de serviços, algumas informações foram sistematizadas no documento elaborado para apoiar discussões sobre atenção à saúde na II Conferência Nacional de Ciência, Tecnologia e Inovação em Saúde (Paim, 2004). Assim, os recursos disponíveis na infra-estrutura do sistema de serviços de saúde apresentam uma distribuição desigual entre regiões, estados e municípios, áreas urbanas e rurais e, nas cidades, entre periferia e centro, além de disparidades no acesso de estratos sociais e raciais.

Enquanto o País possuía em média 3,o leitos disponíveis para o SUS (sendo 2,1 privados) e 1,4 médico por mil habitantes, a região Norte apresentava os seguintes indicadores: 2,1 leitos SUS, 1,2 leito privado e o,6 médico por mil habitantes, correspondendo a cerca da metade dos valores exibidos pela região Sudeste. No caso dos odontólogos, a região Sudeste e Centro-Oeste exibem valores quatro vezes superiores ao Norte e duas vezes ao Nordeste. (Nunes e col., 2001).

No caso dos leitos de UTI disponíveis para o SUS, a média nacional era 6,8 leitos por 100.ooo habitantes enquanto a maioria dos estados da região Norte e Nordeste apresentavam valores inferiores ao nacional, com destaque negativo para Rondônia $(0,5)$ e Bahia $(1,3)$. Quanto à cobertura de DPT, cujo cálculo foi restrito à vacinação de rotina no sentido de refletir o acesso à atenção básica, as medianas desse indicador na região Sul e Sudeste superaram o valor nacional, enquanto os valores médios regionais e estaduais são comparados àqueles observados nos países desenvolvidos. Finalmente, no que diz respeito ao indicador de cobertura de procedimentos de alta complexidade (TRS), verificou-se no ano 2000 uma taxa de 32,o por 100.ooo habitantes para o País, ainda que a utilização esperada, segundo o Ministério da Saúde, devesse ser de 40,o por 100.00o. As taxas de todos os estados da região Sul e Sudeste estão próximas ou acima da média nacional, porém as demais regiões apresentam valores muito baixos desse indicador, a ponto de o
Sudeste atender cinco vezes mais pacientes do que o Norte (Duarte e col., 2002).

Estas desigualdades na oferta de recursos de saúde reproduzem-se no consumo diferenciado de serviços de saúde (Neri e Soares, 2002). Cinco indicadores de acesso/utilização dos serviços de saúde, com periodicidade anual, têm sido utilizados no Brasil: internações hospitalares SUS/10o habitantes, consultas médicas SUS/habitante, cobertura vacinal DPT $\left(3^{\text {a }}\right.$ dose), cobertura vacinal sarampo ( $1^{\text {a }}$ dose) e cobertura vacinal poliomielite ( $3^{\text {a }}$ dose). Assim, em 1999, o País produziu 7,19 internações SUS por 100 habitantes, 2,19 consultas/habitante e coberturas vacinais de 94,6\% (DPT), 99,5\% (AS) e 99,1\% (AP) (Nunes e col., 2001). A análise das desigualdades geográficas e sociais na utilização de serviços de saúde indica uma taxa geral de utilização de 19,49 por 100 mil habitantes para a região Sudeste e 13,01 na região Nordeste em 1989, antes da implantação do SUS. Com a implementação do SUS ocorreu uma redução dessa disparidade pois em 1996/1997 as diferenças entre tais regiões deixaram de ser estatisticamente significantes (Travassos e col., 200o).

Ao se analisarem essas taxas de utilização para os grupos com e sem morbidade, constatam-se diferenças significativas desfavoráveis ao Nordeste. Quando se introduz na análise a renda, como proxy das condições sociais, verifica-se uma tendência de diminuição do acesso aos serviços de saúde, penalizando os mais pobres de ambas as regiões. O percentual de busca de atendimento nos últimos trinta dias por quintil de renda cresce progressivamente de $47,2 \%$ no primeiro para $68,9 \%$ no quinto quintil (Reis, 2001).

No caso da cobertura hospitalar, verifica-se que a região Sudeste exibe uma menor taxa de internação pelo SUS. No que diz respeito às consultas médicas, constata-se um gradiente crescente, acompanhando o desenvolvimento das regiões, embora a região Sul apresente valor inferior àquele verificado no CentroOeste (Nunes e col., 2001).

A variável renda também influi na atenção à saúde e no acompanhamento médico. Embora a existência de problema crônico de saúde $(15,4 \%$ da população) não varie tanto entre os estratos de renda (12,3\% entre os mais pobres e $16,6 \%$ entre os mais ricos), as diferenças se revelam na assistência. No primeiro 
quintil (mais pobres) 54,7\% dos indivíduos são acompanhados por médicos, enquanto no quinto quintil (mais ricos) 82,9\% dos portadores de doenças crônicas dispõem de acompanhamento médico. Estes últimos também realizaram exames periódicos com maior freqüência (Reis, 2001).

Outros motivos de procura de serviços de saúde também guardam uma relação com a renda. Enquanto os de menor renda apresentavam um indicador de $7,0 \%$, os de maior renda praticamente correspondiam ao dobro (13,5\%). Dois serviços revelam muito bem tais discrepâncias: checkup, que variou entre 13,9\% e $28,5 \%$, e odontologia, cuja procura passou de $1,8 \%$ no primeiro quintil para $12,6 \%$ no quinto quintil (Reis, 2001).

Em outro estudo sobre desigualdades na atenção à saúde foram utilizadas, além do número de médicos e de leitos hospitalares por mil habitantes, a razão de leitos de UTI vinculados ao SUS, a percentagem de municípios que atingiram a meta de cobertura da vacina tríplice (DPT) e a proporção de pacientes atendidos por terapia renal substitutiva (TRS). Assim a cobertura de DPT poderia indicar a situação dos procedimentos de baixa complexidade, enquanto a TRS representaria um indicador de cobertura de procedimentos de alta complexidade, envolvendo hemodiálise e transplante renal (Duarte e col., 2002).

No caso da atenção pré-natal, mesmo em um município da Região Metropolitana de São Paulo em que não se verificaram segmentos populacionais excluídos no sistema de saúde - o que sugere a capacidade de inclusão do SUS, observou-se que o estrato com piores condições de vida teve maiores dificuldades de acesso e deficiências quanto à qualidade da atenção (Puccini e col., 2003).

Essas disparidades regionais e particularidades examinadas no presente tópico, além de apontarem possíveis relações entre as desigualdades de saúde e as iniqüidades sociais (Neri e Soares, 2002), revelam “a multiplicidade de fatores que interferem no padrão de consumo de serviços de saúde e a imbricação perversa entre eles no Brasil, resultando em um quadro de desigualdades cumulativas que evidenciam o quão distante encontra-se o sistema de saúde do país dos princípios igualitários enunciados na sua formulação" (Travassos e col. 200o, p.143).

\section{Redução de Desigualdades ou Promoção da Eqüidade no SUS?}

Diversas iniciativas têm procurado reduzir as desigualdades e iniqüidades no SUS. Assim, o redirecionamento de recursos entre regiões do país tem sido uma das medidas adotadas. A implantação do PAB permitiu que o vetor da distribuição de recursos do próprio SUS que privilegiava regiões, estados e municípios dispondo de melhor infra-estrutura passasse a levar em conta a população existente em cada território. Tem-se constatado a redução das desigualdades nos valores alocados pelo gestor federal (Souza, 2003) e na distribuição dos tetos financeiros da assistência à saúde entre as regiões, além da ampliação do acesso à assistência e o aumento da cobertura de imunizações das crianças (Negri, 2002). Entre 1995 e 1999, o número de consultas médicas por habitante cresceu em todas as regiões, de modo que o valor médio nacional passou de 1,91 para 2,19 (Nunes e col., 2001). Esta tendência pode estar expressando a política de prioridade para a atenção básica e a tentativa de reversão do modelo assistencial "hospitalocêntrico".

A combinação de critérios técnicos na alocação (Lima e col., 2002), o desenvolvimento de metodologias para a análise das desigualdades em saúde (Szwarcwald e col.., 2002) e a realização de investigações em política e economia da saúde (Junqueira e col., 2002; Porto, 2002) constituem contribuições para a formulação de políticas de saúde orientadas para a eqüidade. A produção de metodologias e de indicadores para monitorar as desigualdades de saúde e de condições de vida pode ser uma das contribuições das universidades, centros de pesquisa e sistemas de vigilância da saúde no sentido de orientar a formulação e implementação de políticas públicas que reduzam as iniqüidades (Mello-Jorge e col., 2001; Nunes e col., 2001; Duarte e col., 2001).

Assim, algumas evidências apontam para uma discreta redução das desigualdades na distribuição de recursos do SUS (Almeida e col.., 2002; Negri, 2002; Brasil, 2002a), embora ainda se faça necessária uma redistribuição geográfica de recursos financeiros em favor das regiões Norte e Nordeste (Porto, 2002). Alguns receiam, porém, "que as fórmulas adotadas tornem-se complexas e de difícil compreensão, podendo 
não trazer ganhos reais no âmbito da eqüidade" (Cazelli e col., 2002).

No Brasil, os esforços para reduzir as iniqüidades em saúde (Luchesi, 2003; Souza, 2003) consideram a eqüidade na dotação de recursos financeiros, no acesso a serviços de saúde e nos resultados. Distinguemse, ainda, as desigualdades no estado de saúde que expressam desigualdades sociais de modo que a igualdade no acesso aos serviços de saúde não é suficiente, ainda que necessária, para reduzir as desigualdades no adoecer e morrer entre os distintos grupos sociais. Esta distinção tem sido considerada importante na formulação de políticas públicas na perspectiva de trabalhar "com a noção de necessidades diferentes entre distintos grupos sociais, determinadas tanto pela diversidade (sexo, idade, raça) como pela injustiça social (renda, acesso a bens e serviços) ou mesmo por características culturais e subjetivas de grupos e indivíduos" (Pelegrini e col., 2005:277).

Desse modo, diversas propostas metodológicas e técnicas têm sido desenvolvidas na perspectiva dessa eqüidade, insuficientemente teorizada, que no final das contas remetem para a focalização nos mais necessitados (Kadt e Tasca, 1993). Multiplicam-se linhas de pesquisa, abordagens quali-quantitativas e indicadores para mapear desigualdades em saúde nos territórios, na expectativa de que "ao valor moral da eqüidade se incorpore um instrumental capaz de dar objetividade às análises e proposições para a gestão do sistema de saúde" (Pinheiro e col., 2005:451).

Nessa perspectiva, pode ser mencionada como exemplo uma metodologia adotada pelo governo do Rio Grande do Sul que fundamentou uma política denominada Municipalização Solidária da Saúde (MSS). Ao considerar insuficiente a distribuição de recursos para os municípios tomando como critério a população (per capita), privilegiou o Índice de Desenvolvimento Humano (IDH) ao lado do tamanho da população: coeficiente da população total do município (C1); coeficiente da população com idade menor de 14 anos (C2); coeficiente da população com idade maior de 60 anos $\left(\mathrm{C}_{3}\right)$; coeficiente inverso da capacidade instalada municipal (C4); coeficiente ponderado da mortalidade infantil do município ( $\mathrm{C}_{5}$ ); coeficiente do inverso da receita municipal (C6); coeficiente do percentual de recursos próprios utilizados em saúde $\left(\mathrm{C}_{7}\right)$; coeficiente calculado mediante divisão do peso atribuído à ordem de prioridade dada à saúde no orçamento participativo estadual (C8). A partir da adoção desses critérios obteve-se um coeficiente geral com a seguinte fórmula:

\section{$\mathrm{CG}=0,3(\mathrm{CI})+0,1\left(\mathrm{C}_{2}\right)+0,1\left(\mathrm{C}_{3}\right)+0,05\left(\mathrm{C}_{4}\right)+0,05\left(\mathrm{C}_{5}\right)$ $+0,05(\mathrm{C6})+0,05\left(\mathrm{C}_{7}\right)+0,3(\mathrm{C} 8)$.}

Tendo em conta o ano de 2001, foi verificada uma desconcentração de recursos para os municípios em piores condições sócio-econômicas. Ainda assim as autoras recomendam ao final do artigo um aprofundamento da discussão conceitual (Pelegrini e col., 2005).

Nessa experiência reconhecia-se que o grande desafio posto para a gestão seria o de desenvolver mecanismos de alocação de recursos que compatibilizassem eficiência e eqüidade no financiamento das políticas e ações de saúde. Conseqüentemente, o seu propósito fundamental era promover eqüidade no SUS estadual, tomando como referência o artigo 35 da Lei $8080 / 90$. Ainda que tal política seja redistributiva isto não significa que seja mais eqüitativa (Cohn, 2005), sobretudo tendo em conta os baixos valores envolvidos nas transferências e diante do fato de que "a descentralização nada molda, antes se amolda à realidade histórica determinante da sua implantação" (Elias, 2005:290). Segundo este autor, se o conceito de eqüidade não é bem estabelecido torna-se difícil a sua operacionalização. Mesmo assim, o caso gaúcho ilustra um esforço no sentido de tornar o SUS uma política pública inspirada na equidade, ou melhor, no primado da justiça com atenção voltada para as desigualdades sociais.

Entretanto, "a construção de um sistema de saúde como o SUS na periferia do capitalismo e em países com a dimensão e grau de desigualdade como vigente no Brasil, não se constitui em linha reta, mas supõe idas e vindas com inúmeras tentativas" (Elias, 2005: 291-292). Diante das especificidades da federação brasileira, a promoção da eqüidade via SUS passa pelos governos federal, estaduais e municipais, seja por medidas de competência exclusiva de cada um desses níveis, seja pela atuação combinada, compartilhada e solidária desses entes federativos (Ribeiro, 2005).

O SUS, enquanto política pública, pode se beneficiar de acúmulos de experiências como a gaúcha, bem como as resultantes de modelos de atenção alternativos que reduzam as desigualdades, não só no repasse de recursos, mas no controle de danos, riscos e deter- 
minantes bem como na cobertura, acesso, acolhimento, utilização e qualidade dos serviços de saúde. Assim, é no nível local que tais políticas se efetivam, ou seja, o que acontece "na ponta" (Cohn, 2005) utilizando critérios epidemiológicos na análise da situação de saúde (Paim, 2003a; Ribeiro, 2005). Todavia, tanto os critérios de repasse financeiro quanto os epidemiológicos são apenas bases argumentativas para "um processo de negociação política que implique a representação de interesses das distintas esferas de governo" (Elias, 2005:291) e, sobretudo, das pessoas e grupos afetados por tais políticas.

Este planejamento necessário poderá explicitar na esfera pública as diferentes concepções de igualdade e de eqüidade, possibilitando que os atores sociais intervenham no processo político de definição de prioridades e de alocação de recursos, tomando como referência a idéia de justiça.

\section{Conclusões}

Dada a natureza e a complexidade da pergunta inicialmente formulada que orientou as reflexões do presente texto, as conclusões são obviamente parciais e provisórias. A própria ambigüidade do termo eqüidade, as diferentes concepções do SUS presentes no processo político de saúde e as diversificadas formas de sua apresentação em municípios e estados brasileiros, mais ou menos próximas aos dispositivos legais e ao projeto da Reforma Sanitária, impõem que a resposta seja "em termos". Ou seja, o SUS enquanto política pública, foi formulado na perspectiva do acesso universal, da igualdade e da justiça social no que diz respeito às necessidades de saúde da população brasileira. Na medida em que a eqüidade seja concebida na perspectiva ética e da justiça, esta política pública tem um grande potencial de alcançá-la, não obstante as históricas iniqüidades presentes na sociedade brasileira, agravadas pelas novas versões do capitalismo em tempos de globalização.

De qualquer modo, trata-se de uma expressiva política pública de inclusão, já que desde a transição realizada pelas AIS e o SUDS foram incorporados milhões de brasileiros no sistema público de saúde. Estima-se que um terço da população foi incluído no SUS a partir da Constituição de 1988. Programas como o PACS/PSF, além de ampliarem a cobertura de serviços, têm possibilitado reorientações do sistema no sentido da integralidade e da eqüidade.

Ao atender necessidades de saúde de contingentes expressivos da população anteriormente excluídos do sistema, é possível atribuir ao SUS a redução de desigualdades no acesso aos serviços o que, de certo modo, torna a atenção menos iníqüa, embora ainda muito distante do seu ideário igualitário. Algumas evidências sustentam a afirmação anterior, particularmente quando se consideram as grandes regiões do país (Almeida e col., 2002; Negri, 2002; Brasil, 2002; Souza, 2003). Estados e municípios que têm alcançado certa estabilidade político-administrativa, ao lado de um compromisso com valores e proposições presentes no movimento da Reforma Sanitária Brasileira (Junqueira e col., 2002; Viana e col., 2002; Pelegrini e col., 2005), conseguem demonstrar que o SUS, enquanto política pública, pode promover a equidade no sentido de justiça, sem comprometer o seu caráter universal e igualitário.

\section{Referências}

ALMEIDA, C. Saúde e eqüidade nas reformas contemporâneas. Saúde em Debate, Rio de Janeiro, v. 54, p. 621, 2000 .

ALMEIDA, C. Reforma de sistemas de servicios de salud y equidad en América Latina y el Caribe: algunas lecciones de los años 80 y 9o. Cadernos de Saúde Pública, Rio de Janeiro, v. 18, n. 4, p. 905-925, 2002.

ALMEIDA, P. F.; GIOVANELA, L.; MATTOS, M. Sistema de saúde brasileiro: dilemas da universalização. Saúde em Debate, Rio de Janeiro, v. 26, n. 61, p.137-154, 2002.

ANDRADE, E.I.G. et al. Análise da situação da economia da saúde no Brasil: perspectivas para a estruturação de um centro nacional de informações. Belo Horizonte: COOPMED, 2004. 153p.

BELMARTINO, S. Los valores vinculados a equidad en la reforma de la atención médica en Argentina. Cadernos de Saúde Pública, Rio de Janeiro, v. 18, n. 4, p. 10671076, 2002.

BRASIL. Ministério da Saúde. Secretaria de Assistência à Saúde. Reduzindo as desigualdades e ampliando o acesso à assistência à saúde no Brasil, 1998-2002. Brasília, DF, 2002. 28op. 
BUSTAMANTE, F.; PORTALES, C. Evaluación de políticas y programas de salud. In: OPS/CLAD. Políticas de salud en América Latina: aspectos institucionales de su formulación, implementación y evaluación. Caracas, 1988. 421p. p. 79-120.

CARDOSO, A. M.; SANTOS, R.V.; COIMBRA JR., C. E. A. Mortalidade infantil segundo raça/cor no Brasil: o que dizem os sistemas nacionais de informação?. Cadernos de Saúde Pública, Rio de Janeiro, v. 21, n. 5, p.16021608,2005 .

CAZELLI, C.M; BRITO, J. da S.; SÁ, P.K. de; ORMELLI, C.B. Análise das desigualdades regionais na oferta de serviços de saúde: pesquisa da assistência médico-sanitária, 1992 a 1999. Saúde em Debate, Rio de Janeiro, v. 26, n. 61, p. 198-209, 2002.

CHOR, D. ; LIMA, C. R. de A. Aspectos epidemiológicos das desigualdades raciais em saúde no Brasil. Cadernos de Saúde Pública, Rio de Janeiro, v. 21, n. 5, p.15861594, 2005 .

COHN, A. Eqüidade, saúde e critérios para alocação de recursos. Ciência \& Saúde Coletiva, Rio de Janeiro, v. 10, n. 2, p. 287-288, 2005.

CONSELHO NACIONAL DE SAÚDE. Desenvolvimento do sistema Único de saúde no Brasil: avanços, desafios e reafirmação de Princípios e Diretrizes. Saúde em Debate, Rio de Janeiro, v. 26, n. 62, p. 295-312, 2002.

CORDEIRO, H. Descentralização, universalidade e eqüidade nas reformas da saúde. Ciência \& Saúde Coletiva, Rio de Janeiro, v. 6, n. 2, p. 319-328, 2001.

COSTA, N.R. Política social e ajuste macroeconômico. Cadernos de Saúde Pública, Rio de Janeiro, v. 18, p. 1321, 2002. Suplemento.

DUARTE, E. C. et al. Epidemiologia das desigualdades em saúde no Brasil: um estudo exploratório. Brasília: Organização Pan-Americana da Saúde, 2002. 123p.

ELIAS, P. E. A utilização da noção de eqüidade na alocação de recursos em tempos do pensamento (neo)liberal: anotações para o debate. Ciência \& Saúde Coletiva, Rio de Janeiro, v. 10, n. 2, p. 289-292, 2005.

ESCOREL, S. Reviravolta da saúde: origem e articulação do movimento sanitário. Rio de Janeiro: Editora FIOCRUZ, 1998. p. 175-206.
FAERSTEIN, E. Fórum: raça, racismo e saúde no Brasil. Cadernos de Saúde Pública, Rio de Janeiro, v. 21, n. 5, p. 1584-1585, 2005.

HERINGER, R. Desigualdades raciais no Brasil: síntese de indicadores e desafios no campo das políticas públicas. Cadernos de Saúde Pública, Rio de Janeiro, v. 18, p. 57-65, 2002. Suplemento.

HERNÁNDEZ, M. Reforma sanitaria, equidad y derecho a la salud en Colombia. Cadernos de Saúde Pública, Rio de Janeiro, v. 18, n. 4, p. 991-1001, 2002.

INFANTE, A.; MATA I. de la; LÓPEZ-ACUÑA, D. Reforma de los sistemas de salud en América Latina y el Caribe: situación y tendencias. Revista Panamericana de Salud Pública, Washington, v. 8, n. 1/2, p.13-20, 2000.

JUNQUEIRA, V. et al. Equidad en la salud: evaluación de políticas públicas en Belo Horizonte, Minas Gerais, Brasil, 1993-1997. Cadernos de Saúde Pública, Rio de Janeiro, v. 18, n. 4, p. 1087-1101, 2002.

KADT, E. de; TASCA, R. Promovendo a equidade: um novo enfoque com base no setor da saúde. São Paulo: Hucitec-Cooperação Italiana em Saúde, 1993. 107p.

LABRA, M.E. La reinvención neoliberal de la inequidad en Chile: el caso de la salud. Cadernos de Saúde Pública, Rio de Janeiro, v. 18, n. 4, p. 1041-1052, 2002.

LIMA, J. de C. et al. Desigualdades no acesso e utilização de serviços de saúde no Brasil. Saúde em Debate, Rio de Janeiro, v. 26, n. 6o, p. 62-70, 2002.

LOPES, F. Para além da barreira dos números: desigualdades raciais e saúde. Cadernos de Saúde Pública, Rio de Janeiro, v. 21, n. 5, p. 1595-16o1, 2005.

LUCHESE, P. T. R. Equidade na gestão descentralizada do SUS: desafios para a redução de desigualdades em saúde. Ciência \& Saúde Coletiva, Rio de Janeiro, v. 8, n. 2, p. 439-448, 2003.

MELLO JORGE, M. H. P.; GOTLIEB, S. L. D; LAURENTI, R. A saúde no Brasil: análise do período 1996 a 1999. Brasília: Organização Pan-Americana da Saúde, 2001. p. 27.

MITJAVILA, M.; FERNÁNDEZ, J.; MOREIRA, C. Propuestas de reforma en salud Y equidad en Uruguay: Redefinición del Welfare State?. Cadernos de Saúde Pública, Rio de Janeiro, v. 18, n. 4, p. 1103-1120, 2002. 
NEGRI, B. A política de saúde no Brasil nos anos 1990: avanços e limites. In: NEGRI, B.; D’ÁVILA, A.L. (Org.) $O$ Sistema Único de Saúde em dez anos de desafios. São Paulo: Sobravime, Cealag, 2002. p.15-42.

NERI, M.; SOARES, W. Desigualdade social e saúde no Brasil. Cadernos de Saúde Pública, Rio de Janeiro, v. 18, p. 77-87, 2002. Suplemento.

NORONHA, J.C. de; SOARES, L. T. A política de saúde no Brasil nos anos 9o. Ciência \& Saúde Coletiva, Rio de Janeiro, v. 6, n. 2, p. 445-450, 2001.

NUNES, A.; SANTOS, J.R.S.; BARATA, R.B.; VIANNA, S.M. Medindo as desigualdades em saúde no Brasil: uma proposta de monitoramento. Brasília: Organização Pan-Americana de Saúde, Instituto de Pesquisa Econômica Aplicada, 2001. 224 p.

OPS - Organización Panamericana de la Salud. La cooperación de la Organización Panamericana de la Salud ante los procesos de reforma del sector salud. Washington, 1997. 31p.

OXMAN, G. Evaluación de Políticas de Salud. In: OPS/ CLAD. Políticas de Salud en América Latina. Aspectos institucionales de su formulación, implementación y evaluación. Caracas, 1988. p. 53-78.

PAIM, J. S. A democratização da saúde e o SUDS: o caso da Bahia. Saúde em Debate, Rio de Janeiro, v. 21, p. 3944, 1988.

PAIM, J. S. Saúde, política e reforma sanitária. Salvador: CEPS-ISC, 2002. 446p.

PAIM, J. S. Epidemiologia e planejamento: a recomposição das práticas epidemiológicas na gestão do SUS. Ciência \& Saúde Coletiva, Rio de Janeiro, v. 8, n. 2, p. 557-567, 2003 a.

PAIM, J. S. Gestão da atenção básica nas cidades. In: NETO, E. R.; BÓGUS, C.M. Saúde nos aglomerados urbanos: uma visão integrada. Brasília: Organização PanAmericana da Saúde, 2003b. p.183-212. (Série Técnica Projeto de Desenvolvimento de Serviços de saúde, 3 ).

PAIM, J. S. Políticas de saúde no Brasil. In: ROUQUAYROL, M.Z.; ALMEIDA FILHO, N. Epidemiologia \& Saúde. 6.ed. Rio de Janeiro: Medsi, 2003c. p. 587-6o3.
PAIM, J. S. Atenção à saúde no Brasil. In: BRASIL. Ministério da Saúde. Saúde no Brasil: contribuições para a agenda de prioridades de pesquisa. Brasília, 2004. p.15-44.

PEGO, R.A.; ALMEIDA, C. Teoría y práctica de las reformas en los sistemas de salud: los casos de Brasil y México. Cadernos de Saúde Pública, Rio de Janeiro, v. 18, n. 4, p. 971-989, 2002.

PELEGRINI, M.L.M. de; CASTRO, J.D. de; DRACHLER, M. de L. Eqüidade na alocação de recursos para a saúde: a experiência do Estado do Rio Grande do Sul, Brasil. Ciência \& Saúde Coletiva, Rio de Janeiro, v. 1o, n. 2, p. 275-286, 2005 .

PINHEIRO, M.C.; WESTPHAL, M.F.; AKERMAN, M. Eqüidade em saúde nos relatórios das conferências nacionais de saúde pós-Constituição Federal brasileira de 1988. Cadernos de Saúde Pública, Rio de Janeiro, v. 21, n. 2, p. 449-458, 2005.

PORTO, S. Equidad y distribución geográfica de recursos financeiros en los sistemas de salud. Cadernos de Saúde Pública, Rio de Janeiro, v. 18, n. 4, p. 939-957, 2002.

PUCCINI, R. F. et al. Eqüidade na atenção pré-natal e ao parto em área da Região Metropolitana de São Paulo, 1996; Cadernos de Saúde Pública, Rio de Janeiro, v.19, n. 1, p. 35-45, 2003.

REIS, C. O. O. Desigualdades no acesso aos serviços de saúde. In: NEGRI, B.; DI GIOVANNI, G. (Org.). Brasil: radiografia da saúde. Campinas: UNICAMP, 2001. p. 579-585.

RIBEIRO, P. Distribuição de recursos financeiros e eqüidade: uma relação delicada na saúde. Ciência $\&$ Saúde Coletiva, Rio de Janeiro, v. 10, n. 2, p. 292-296, 2005.

SEN, A. Por que la equidad em salud? Pan American Journal of Public Health, Washington, v. 11, n. 5-6, p.302-309, 2002.

SIQUEIRA-BATISTA; SHRAMM, F. R. A saúde entre a iniqüidade e a justiça: contribuições da igualdade complexa de Amartya Sen. Ciência \& Saúde Coletiva, Rio de Janeiro, v.10, n.1, p.129-142, 2005. 
SOARES, L. T. R. Ajuste neoliberal e desajuste social na América Latina. Rio de Janeiro:UFRJ, 1999. 368p.

SOUZA, R. R. Redução das desigualdades regionais na alocação dos recursos federais para a saúde. Ciência \& Saúde Coletiva, Rio de Janeiro, v. 8, n. 2, p. 449-46o, 2003.

STARFIELD, B. Atenção primária: equilibrio entre necessidades de saúde, serviços e tecnologia. Brasília: UNESCO, Ministério da Saúde, 2002. 725p.

SZWARCWALD, C. L.; BASTOS, F. I.; ANDRADE, C. L. T. Medidas de desigualdad en salud: la discusión de algunos aspectos metodológicos con una aplicación para la mortalidad neonatal en el Município de Rio de Janeiro, 20oo. Cadernos de Saúde Pública, Rio de Janeiro, v. 18, n. 4, p. 959-970, 2002.
TRAVASSOS, C.; VIACAVA, F.; FERNANDES, C.; ALMEIDA, C. M. Desigualdades geográficas e sociais na utilização de serviços de saúde no Brasil. Ciência \& Saúde Coletiva, Rio de Janeiro, v. 5, n. 1, p. 133-149, 2000.

VIANA, A. L. D'A.; HEIMANN, L. S.; LIMA, L. D. et al. Mudanças significativas no processo de descentralização do sistema de saúde no Brasil. Cadernos de Saúde Pública, Rio de Janeiro, v.18, p.139-151, 2002. Suplemento.

WHITEHEAD, M. The concepts and principles of equity and health. Copenhagen: World Health Organization; 1990. 